E D I T O R I A L S

\title{
The ICD in Heart Failure - Time for a Rethink?
}

\author{
John J.V. McMurray, M.D.
}

In a number of primary-prevention trials, implantable cardioverter-defibrillators (ICDs) have been shown to reduce the risk of premature death among patients with left ventricular systolic dysfunction after myocardial infarction and among patients with heart failure and reduced ejection fraction. ${ }^{1}$ The evidence of a benefit is less robust for patients with heart failure who do not have coronary artery disease; the single largest trial, involving 458 such patients, did not show a significant reduction in mortality in association with ICD therapy. ${ }^{2}$ As a result, the current guideline recommendations are based on a meta-analysis of small trials involving patients with nonischemic cardiomyopathy, as well as subgroups with nonischemic cardiomyopathy from larger trials. ${ }^{3}$ Against this background, in the Danish Study to Assess the Efficacy of ICDs in Patients with Non-ischemic Systolic Heart Failure on Mortality (DANISH), the results of which are now reported in the Journal, Køber and colleagues randomly assigned 1116 patients with nonischemic heart failure to receive conventional therapy or an ICD added to conventional therapy and followed them for a median of 5.6 years. ${ }^{4}$ Although ICD therapy was associated with a risk of sudden cardiac death that was half that associated with conventional therapy, the effect on overall mortality, the primary end point of the trial, was not significant.

How should we interpret these findings? That there was no significant benefit with regard to overall mortality in the DANISH trial was a result of the low risk of sudden cardiac death among the patients included in the trial. This low risk reflects two features of the trial. First, patients with nonischemic cardiomyopathy have lower rates of sudden cardiac death and of death from any cause than do patients with an ischemic cause of heart failure. Second, and probably more importantly, the background treatment in the trial was very comprehensive. The use of angiotensin-converting-enzyme inhibitors or angiotensin-receptor blockers and beta-blockers was almost universal, and close to $60 \%$ of patients were treated with a mineralocorticoid-receptor antagonist. In addition, and unlike in previous ICD trials, $58 \%$ of patients received cardiac-resynchronization therapy. Collectively, these treatments reduce the risk of premature cardiovascular death, including the risk of sudden cardiac death, very substantially. ${ }^{1}$ In the control group in the trial, approximately $73 \%$ of all deaths were attributed to a cardiovascular cause, one of the lowest proportions ever described in a trial involving patients with heart failure. ${ }^{5}$ The declining rate of death from cardiovascular causes makes it harder to demonstrate a benefit of additional therapy for heart failure with regard to overall mortality. ${ }^{5}$ This is especially so for ICDs, which modify only one of the two major modes of death, unlike pharmacologic therapies, which reduce the risk of death from worsening heart failure as well as the risk of sudden cardiac death. Consequently, with an ICD, there is not only the risk of death from noncardiovascular causes but also the risk from progressive pump failure and other cardiovascular causes. These competing risks change with time as heart failure advances (and the risk of nonsudden death rises), and noncardiovascular causes of death become more frequent with increasing age and increasing numbers of coexisting conditions. Thus, the combination of a low overall mortality rate and a relatively small proportion of deaths due to cardiovascular causes, with only approximately a third of deaths in the control group occurring suddenly, made it difficult to show a significant benefit of ICD therapy 
with regard to the risk of death from any cause with the number of patients enrolled. Moreover, any such benefit was likely to decline with time, as seems to have been the case.

What are the implications of these findings for clinical practice? The results of the DANISH trial probably represent the most optimistic estimate of the benefit of ICD therapy in patients with nonischemic heart failure who receive evidence-based treatment. Patients enrolled in trials are usually younger and have fewer coexisting conditions than do those in the community and, as a consequence, have a relatively higher risk of death from cardiovascular causes than from noncardiovascular causes. ${ }^{6,7}$ In keeping with this observation, there was a suggestion of a differential response according to age in the present trial, with a possible benefit of ICD therapy with regard to death from any cause among younger patients ( $<68$ years of age). The background rate of death from cardiovascular causes and sudden cardiac death could also be reduced even more in practice by further optimizing pharmacologic therapy, with greater use of mineralocorticoid-receptor antagonists and new treatments shown to reduce risk in heart failure, as well as the use of coronary revascularization in patients with ischemic cardiomyopathy. ${ }^{8,9}$ Consequently, the absolute benefit of ICDs in a typical and well-treated population with heart failure might be small. ICDs are expensive and not without adverse effects. It is, therefore, desirable to avoid their use in patients who are unlikely to obtain a worthwhile benefit. These considerations highlight the need to target ICDs to the patients most likely to benefit - that is, those who remain at high absolute risk for sudden cardiac death despite receiving the best available pharmacologic and device therapy. The results of the DANISH trial, coupled with the generally infrequent use of ICDs globally, should open a debate about whether it is ethical to conduct new ICD trials involving the highest-risk patients. The challenge is how to identify such patients. ${ }^{10}$

Disclosure forms provided by the author are available with the full text of this editorial at NEJM.org.

From the British Heart Foundation Cardiovascular Research Centre, University of Glasgow, Glasgow, United Kingdom.

This editorial was published on August 28, 2016, at NEJM.org.

1. Priori SG, Blomström-Lundqvist C, Mazzanti A, et al. 2015 ESC Guidelines for the management of patients with ventricular arrhythmias and the prevention of sudden cardiac death: the Task Force for the Management of Patients with Ventricular Arrhythmias and the Prevention of Sudden Cardiac Death of the European Society of Cardiology (ESC) - endorsed by: Association for European Paediatric and Congenital Cardiology (AEPC). Eur Heart J 2015;36:2793-867.

2. Kadish A, Dyer A, Daubert JP, et al. Prophylactic defibrillator implantation in patients with nonischemic dilated cardiomyopathy. N Engl J Med 2004;350:2151-8.

3. Desai AS, Fang JC, Maisel WH, Baughman KL. Implantable defibrillators for the prevention of mortality in patients with nonischemic cardiomyopathy: a meta-analysis of randomized controlled trials. JAMA 2004;292:2874-9.

4. Kфber L, Thune JJ, Nielsen JC, et al. Defibrillator implantation in patients with nonischemic systolic heart failure. $\mathrm{N} \mathrm{Engl}$ J Med 2016;375:1221-30.

5. Rush CJ, Campbell RT, Jhund PS, et al. Falling cardiovascular mortality in heart failure with reduced ejection fraction and implications for clinical trials. JACC Heart Fail 2015;3:603-14.

6. Steinberg BA, Al-Khatib SM, Edwards R, et al. Outcomes of implantable cardioverter-defibrillator use in patients with comorbidities: results from a combined analysis of 4 randomized clinical trials. JACC Heart Fail 2014;2:623-9.

7. Hess PL, Al-Khatib SM, Han JY, et al. Survival benefit of the primary prevention implantable cardioverter-defibrillator among older patients: does age matter? An analysis of pooled data from 5 clinical trials. Circ Cardiovasc Qual Outcomes 2015; $8: 179-86$.

8. McMurray JJV, Packer M, Desai AS, et al. Angiotensin-neprilysin inhibition versus enalapril in heart failure. $\mathrm{N}$ Engl J Med 2014;371:993-1004.

9. Velazquez EJ, Lee KL, Jones RH, et al. Coronary-artery bypass surgery in patients with ischemic cardiomyopathy. N Engl J Med 2016;374:1511-20.

10. Pimentel M, Rohde LE, Zimerman A, Zimerman LI. Sudden cardiac death markers in non-ischemic cardiomyopathy. J Electrocardiol 2016;49:446-51.

DOI: $10.1056 /$ NEJMe1609826

Copyright () 2016 Massachusetts Medical Society.

\title{
Antibiotic Prophylaxis for Cesarean Delivery - When Broader Is Better
}

\author{
Robert A. Weinstein, M.D., and Kenneth M. Boyer, M.D.
}

Approximately 4 million babies are born each year in the United States. Of these infants, about a third are delivered by cesarean section. One of the many concerns about cesarean deliveries is the high risk of maternal infectious complications, which are 5 to 10 times more frequent than with vaginal deliveries. ${ }^{1}$ During cesarean delivery, the endometrial cavity and operative field may be seeded 